\section{ATH-04 COMPARING THE DIAGNOSTIC YIELD AND SAFETY OF ENDOSONOGRAPHY GUIDED USE OF SHARK-CORE AND PRO-CORE NEEDLES}

Zia Rahman*, Kashif Riaz, Mohsin Hossein, Sophia Savva, Sudarshan Kadri. Leicester Royal Infirmary, Leicester, UK

\subsection{6/gutjnl-2019-BSGAbstracts. 11}

Background Endoscopic ultrasound(EUS) guided fine needle aspiration and biopsy (EUS-FNA/FNB) is the standard technique for diagnosis and evaluation of pancreatic lesions. Core biopsy specimens provide accurate diagnoses. ProCore needle (Wilson-Cook Medical Inc. NC,USA) and Shark-Core needle (Covidien, Ireland) were designed to obtain histological and cytological samples. Each claim superiority over other for better diagnostic acquisition and safety. There is limited head to head comparison data available in literature about different needles.

Aim The aim of our study was to compare the diagnostic yield and safety of $22 \mathrm{G}$ FNB needles for sampling of pancreatic lesions.

Methods We prospectively randomised patients with pancreatic lesions either to Shark-Core or to Pro-Core needle sampling. Data collection included demographics, needle type, number of passes.

Results A total of 143 patients having 151 pancreatic lesions were analysed.

74 lesions were biopsied in Shark-Core group from 69 patients. Four patients had their procedure repeated. Mean age was 62.4 years and $51 \%$ were males. Mean number of needle passes were four. 33 samples had malignancy and 26 had other diagnoses and 10 had insufficient tissue. The diagnostic yield was $86.3 \%$. 2 patients developed mild pancreatitis and one required admission with epigastric pain and vomiting.

Pro-Core group had 77 lesions biopsied from 74 patients. 3 had two procedures each and one patient had both Shark core and Procore sampling. Mean age was 63 with males 50.8\%. Median passes were four. 37 had malignancy and 23 had other diagnoses. Inadequate tissue sample was obtained in 13. The overall diagnostic yield was $63 / 77(81.81 \%) .3$ patients had epigastric pain and vomiting and needed admission.

Discussion Though Shark-Core needle demonstrated better diagnostic yield and had marginally more complications, both of which were not statistically different from Pro-Core needle. In our experience, the time required for tissue acquisition is in favor of Shark-Core needle as it allows inner needle to be withdrawn than the whole system, maintaining the scope position unlike Pro-Core system that requires the whole biopsy needle system to be withdrawn from the scope.

With many more core sampling needles now commercially available, further studies may be required to evaluate other types of needles for tissues yield, safety as well as time required to acquire samples.

\section{ATH-05 CAN PROTOCOLISED MEASUREMENTS WITH BARIUM RADIOLOGY PREDICT SEVERITY AND TREATMENT OUTCOMES IN ZENKER'S DIVERTICULUM?}

${ }^{1}$ Keith Siau*, ${ }^{1}$ Akhmid Aziz, ${ }^{1}$ Adrian Hall, ${ }^{1}$ Min Lee, ${ }^{2}$ Hamid Mohaghegh, ${ }^{1}$ Linzie Priestnall, ${ }^{1}$ Andrea Gait, ${ }^{3}$ Chris I Mulder, ${ }^{1}$ Sauid Ishaq. ${ }^{1}$ Dudley Group Hospitals NHSFT, Dudley, UKi ${ }^{2}$ Shahid Beheshti University of Medical Sciences, Tehran, Iran; ${ }^{3} \mathrm{VU}$ University Medical Center, Amsterdam, Netherlands

\subsection{6/gutinl-2019-BSGAbstracts. 12}

Introduction Barium swallow is an established investigation for Zenker's diverticulum (ZD), yet, no agreed measurement protocol exists for the evaluation of $\mathrm{ZD}$ on Barium radiology. We developed a standardised protocol to measure ZD dimensions on Barium radiology, and aimed to correlate $\mathrm{ZD}$ dimensions with symptoms, procedural difficulty and treatment outcomes.
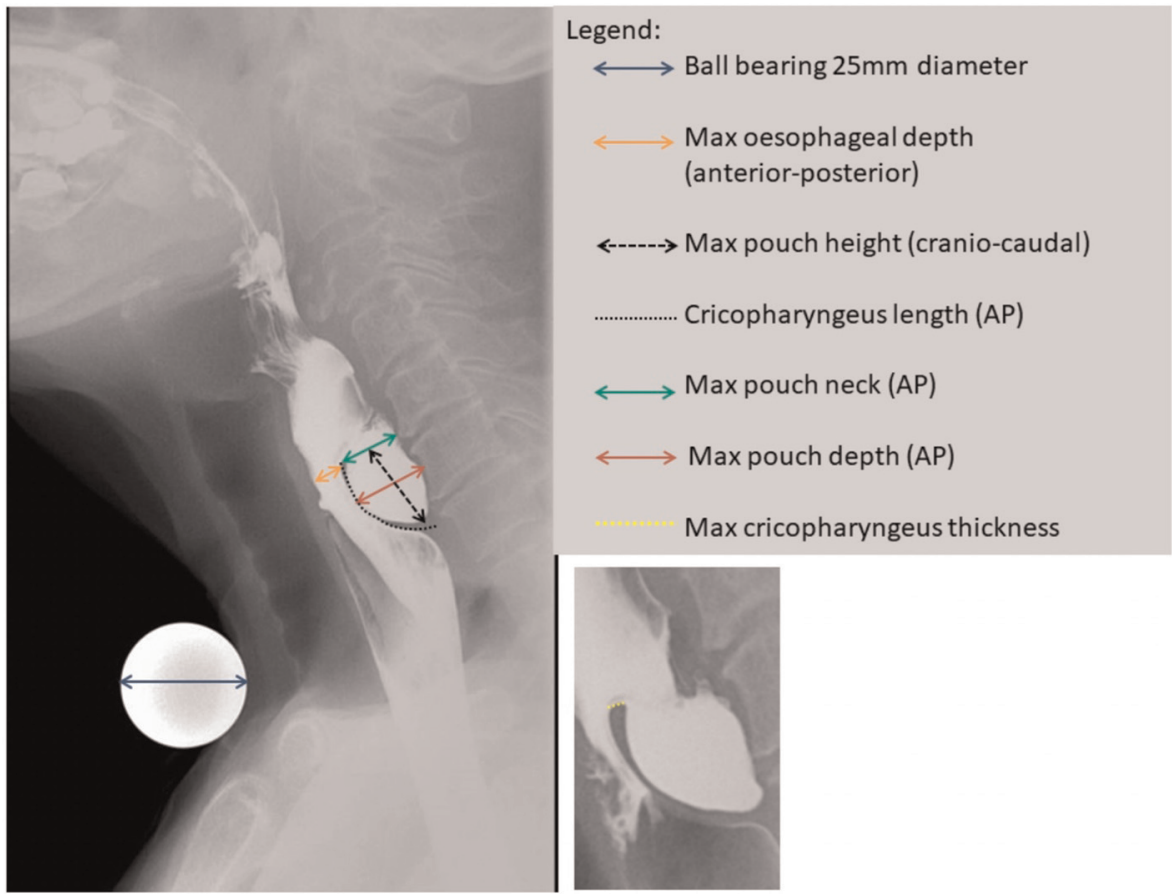
Methods This prospective single-centre study included patients with symptomatic ZD undergoing flexible endoscopic septal division (FESD) as day case procedures between 2014-2018. Patients underwent Barium swallow imaging; ZD dimensions (figure 1) were measured and agreed by two expert radiologists using a predefined protocol. Symptom severity pre- and post-FESD was recorded using the Dysphagia, Regurgitation, Complications (DRC) scale. ${ }^{1}$ Procedural difficulty was rated on a three-point scale: easy, moderate, difficult. The primary outcome was therapeutic success, defined as remission following single episode FESD with a DRC score of 1 or less art 6 months of follow-up. ZD dimensions were subjected to MannWhitney tests and logistic regression analyses.

Results In total, 68 patients (mean age 74.2, SD 11.8) underwent barium radiology. Male gender comprised $60.9 \%$ of the cohort and was associated with larger pouch height ( $\mathrm{P}=0.008)$, width $(\mathrm{P}=0.004)$ and depth $(\mathrm{P}=0.045)$. A positive correlation was identified between baseline DRC score and pouch depth (rho 0.319, $\mathrm{P}=0.012$ ), particularly the symptom of regurgitation $(\mathrm{P}=0.009)$. No significant associations were found between ZD dimensions and procedural difficulty. Overall, each patient underwent an average of 1.4 FESD procedures. The outcome of therapeutic success at 6-months was achieved in 69\% and was associated with shorter pouch height (median $14.5 \mathrm{~mm}$ vs. $19 \mathrm{~mm}, \mathrm{P}=0.030$ ) and pouch width (median $20 \mathrm{~mm}$ vs. $28 \mathrm{~mm}, \mathrm{P}=0.046$ ), with smaller cricopharyngeal length tending towards significance (median 20.8 $\mathrm{mm}$ vs. $26.3 \mathrm{~mm}, \mathrm{P}=0.051$ ). On multivariable analysis using a forward stepwise approach, pouch height was the sole dimension affecting the study outcome, with each additional $\mathrm{mm}$ in pouch height associated with a decrement in the probability of therapeutic success (OR 0.946, 95\% CI: 0.897-0.997, $\mathrm{P}=0.031$ ).

Conclusions ZD dimensions may be feasibly evaluated using Barium radiology. Specific parameters, especially those relating to the pouch, appear to correlate with baseline severity and post-FESD patient outcomes. These results may inform the planning of FESD in day case patients with $\mathrm{ZD}$.

\section{REFERENCE}

1. Battaglia G, Dig Endosc 2015. PMID: 25975384.

\section{ATH-06 FIRST CLINICAL EXPERIENCE WITH SPEEDBOAT RS2, A NEW MULTIMODALITY DEVICE FOR COLONIC SUBMUCOSAL DISSECTION}

\footnotetext{
1,2Zacharias P Tsiamoulos*, ${ }^{2}$ loannis Stasinos, ${ }^{1}$ Aristeidis Oikonomakis, ${ }^{1} J o s e p h$ Sebastian, ${ }^{1}$ Nipin Bagla, ${ }^{3}$ Christopher Hancock, ${ }^{2}$ Brian P Saunders. ${ }^{1}$ East Kent Hospitals University, Margate, UK; ${ }^{2}$ St Mark's Hospital, London, UK; ${ }^{3}$ Bangor University, UK
}

\subsection{6/gutjnl-2019-BSGAbstracts.13}

Introduction The 'Speedboat-RS2' (Creo-Medical/Wales/UK) is the first multi-modality device, incorporating bipolar-radiofrequency energy for cutting, microwave energy for coagulation, an integrated needle for submucosal injection, and a rotatable blade with a heat insulated hull.

Methods A prospective database from June 2018 to Jan 2019 was analyzed including 20 consecutive patients (mean age: $69.7 \pm 15.18$ years, $50 \%$ males) with 20 colorectal Lateral Spreading Tumours/LSTs. Two operators performed all colonic endoscopic submucosal dissections/ESDs.

Results Sixteen procedures were performed under conscious sedation and 4 general anaesthesia (Olympus/CF-HQ290ZL/
$13.2 \mathrm{~mm}$ tip diameter). Median lesion size was $4 \mathrm{~cm}$ (range 1-9 cm). The LSTs and procedural characteristics are described in table 1 .

\begin{tabular}{|c|c|c|c|c|c|c|}
\hline Case & Polyp site & $\begin{array}{l}\text { Polyp } \\
\text { Morphology }\end{array}$ & Procedure & $\begin{array}{l}\text { Procedure } \\
\text { Time (hrs) }\end{array}$ & $\begin{array}{l}\text { Polyp size } \\
\text { (cm) }\end{array}$ & $\begin{array}{l}\text { Delayed } \\
\text { Bleeding }\end{array}$ \\
\hline 1 & SC & $\begin{array}{l}\text { IST-G } \\
\text { (MN) }\end{array}$ & $\begin{array}{l}\text { ESD/snare } \\
\text { conversion }\end{array}$ & $>2$ & 6 & No \\
\hline 2 & SC & $\begin{array}{l}\text { LST-G } \\
\text { (MN) }\end{array}$ & $\begin{array}{l}\text { ESD/snare } \\
\text { conversion }\end{array}$ & $1-2$ & 7 & No \\
\hline 3 & Rectum & $\begin{array}{l}\text { LST-NG } \\
\text { (F) }\end{array}$ & $\begin{array}{l}\text { ESD } \\
\text { En-bloc }\end{array}$ & $<1$ & 1 & No \\
\hline 4 & SC & $\begin{array}{l}\text { LST-G } \\
(U)\end{array}$ & $\begin{array}{l}\text { ESD } \\
\text { En-bloc }\end{array}$ & $<1$ & 4 & No \\
\hline 5 & Rectum & $\begin{array}{l}\text { LST-G } \\
\text { (U) }\end{array}$ & $\begin{array}{l}\text { ESD/snare } \\
\text { conversion }\end{array}$ & $1-2$ & 5 & No \\
\hline 6 & Caecum & $\begin{array}{l}\text { IST-G } \\
\text { (U) }\end{array}$ & $\begin{array}{l}\text { Abandoned } \\
\text { 'Muscle } \\
\text { retraction sign' }\end{array}$ & NA & 3 & NA \\
\hline 7 & $\mathrm{AC}$ & $\begin{array}{l}\text { LST-NG } \\
\text { (F) }\end{array}$ & $\begin{array}{l}\text { ESD/snare } \\
\text { conversion }\end{array}$ & $1-2$ & 4 & No \\
\hline 8 & $\begin{array}{l}\text { Hepatic } \\
\text { flexure }\end{array}$ & $\begin{array}{l}\text { LST-G } \\
\text { (U) }\end{array}$ & $\begin{array}{l}\text { ESD } \\
\text { En-bloc }\end{array}$ & $1-2$ & 4 & No \\
\hline 9 & SC & $\begin{array}{l}\text { IST-G } \\
(U)\end{array}$ & $\begin{array}{l}\text { ESD } \\
\text { En-bloc }\end{array}$ & $1-2$ & 3 & No \\
\hline 10 & Rectum & $\begin{array}{l}\text { LST-G } \\
\text { (MN) }\end{array}$ & $\begin{array}{l}\text { ESD/snare } \\
\text { conversion }\end{array}$ & $>2$ & 4.5 & Yes \\
\hline 11 & TC & $\begin{array}{l}\text { LST-NG } \\
\text { (F) }\end{array}$ & $\begin{array}{l}\text { ESD/snare } \\
\text { conversion }\end{array}$ & $>2$ & 9 & Yes \\
\hline 12 & $\mathrm{AC}$ & $\begin{array}{l}\text { LST-G } \\
(U)\end{array}$ & $\begin{array}{l}\text { ESD/snare } \\
\text { conversion }\end{array}$ & $1-2$ & 4.5 & No \\
\hline 13 & SC & $\begin{array}{l}\text { LST-G } \\
(U)\end{array}$ & $\begin{array}{l}\text { ESD } \\
\text { En-bloc }\end{array}$ & $<1$ & 2.5 & No \\
\hline 14 & RS & $\begin{array}{l}\text { LST-G } \\
\text { (U) }\end{array}$ & $\begin{array}{l}\text { ESD } \\
\text { En-bloc }\end{array}$ & $1-2$ & 4 & No \\
\hline 15 & Caecum & $\begin{array}{l}\text { LST-NG } \\
\text { (F) }\end{array}$ & $\begin{array}{l}\text { ESD/hybrid } \\
\text { En-bloc }\end{array}$ & $1-2$ & 3.5 & No \\
\hline 16 & TC & $\begin{array}{l}\text { IST-G } \\
(\mathrm{U})\end{array}$ & $\begin{array}{l}\text { ESD } \\
\text { En-bloc }\end{array}$ & $<1$ & 3.6 & No \\
\hline 17 & Rectum & $\begin{array}{l}\text { LST-NG } \\
\text { (F) }\end{array}$ & $\begin{array}{l}\text { ESD/snare } \\
\text { conversion }\end{array}$ & $<1$ & 5 & No \\
\hline 18 & Rectum & $\begin{array}{l}\text { LST-G } \\
(U)\end{array}$ & $\begin{array}{l}\text { ESD } \\
\text { En-bloc }\end{array}$ & $<1$ & 4 & No \\
\hline 19 & $\mathrm{AC}$ & $\begin{array}{l}\text { LST-G } \\
(U)\end{array}$ & $\begin{array}{l}\text { ESD/hybrid } \\
\text { En-bloc }\end{array}$ & $<1$ & 2.5 & No \\
\hline 20 & Rectum & $\begin{array}{l}\text { LST-G } \\
\text { (MN) }\end{array}$ & $\begin{array}{l}\text { ESD } \\
\text { En-bloc }\end{array}$ & $1-2$ & 3.5 & Yes \\
\hline
\end{tabular}

1/20 case was abandoned during dissection. Endoscopically en-bloc resection was achieved in 11/19 (57.8\%) cases and 10/ 19 cases (52.6\%) resections were R0 histologically. In 8/19 patients snare excision was used to facilitate resection with a median of 2 resection pieces. Microwave energy was utilized to pre-coagulate and control active bleeding successfully in 17/ $19(89.5 \%)$ cases and haemostatic monopolar forceps was required in 2 cases. Histology showed dysplasia in 15/19, NET in $1 / 19$ and T1 cancer in 3/19. 2/3 patients with T1 cancers had endoscopic surveillance with no recurrence and 1 had surgery and no residual tissue. 3/19 patients experienced 